\title{
Reconstruction of 3D stress fields basing on piezooptic experiment
}

\author{
Vasylkiv Yu. ${ }^{1}$, Kvasnyuk O. ${ }^{1}$, Krupych O. ${ }^{1}$, Mys O. ${ }^{1}$, \\ Maksymuk O. ${ }^{2}$ and Vlokh R. ${ }^{1}$ \\ ${ }^{1}$ Institute of Physical Optics of the Ministry of Education and Science of \\ Ukraine, 23 Dragomanov St., 79005 Lviv, Ukraine, e-mail:vlokh@ifo.lviv.ua \\ ${ }^{2}$ Ya. S. Pidstryhach Institute of Applied Mechanics and Mathematics of the Na- \\ tional Academy of Sciences of Ukraine, 3b Naukova St., 79060 Lviv, Ukraine
}

Received: 9.01.2009

\begin{abstract}
We suggest a technique for reconstructing 3D stress tensor fields with using polarimetric mapping, optical retardation simulations on the basis of integral Jones matrix approach and a relevant mechanical model. It is shown that the reconstruction of stress fields essentially increases the accuracy of piezooptic experiments, while the corresponding errors could be reduced almost to an apparatus error.
\end{abstract}

Keywords: piezooptics, stress tensor field reconstruction, optical retardation simulation, experimental error

PACS: $78.20 . \mathrm{Hp}$, 07.10.Lw, 07.60.Fs

UDC: 535.012 .2

\section{Introduction}

Piezooptic effect has been known as long ago as beginning from the first studies carried out by Brewster in 1818. This phenomenon is utilized in different technical branches, in particular for designing optical stress sensors and devices that measure acceleration, in stress field tomography, etc. (see, e.g., [1-4]). Besides, the piezooptic (or photoelasic) effect is to be properly taken into account when designing electrooptic devices. Far more important, the photoelasic effect underlies acoustooptic phenomena which are widely explored [5-8]. Therefore the knowledge of accurate values of piezooptic parameters of materials used in different practical applications is of a great importance.

As a matter of fact, the piezooptic coefficients $\left(\pi_{\lambda \mu}\right)$ figuring in the relation

$$
\Delta B_{\lambda}=\pi_{\lambda \mu} \sigma_{\mu}
$$

(with $\Delta B_{\lambda}$ being the increment of optical-frequency impermeability tensor and $\sigma_{\mu}$ the mechanical stress components where $\lambda, \mu=1,2,3,4,5,6$ correspond to $11,22,33,32,31,21$ under transforming the matrix notation to the tensor one) are usually measured with rather high errors, which often exceed tens of per cents (of many works demonstrating this fact see, e.g., [9-12]). On the contrary, the photoelastic coefficients ( $p_{\lambda \nu}=\pi_{\lambda \nu} C_{\nu \mu}$, where $C_{\nu \mu}$ are the elastic stiffness tensor) could be determined with higher accuracy with the known 
Dixon method $[13,14]$. However, recalculations of the photoelastic coefficients to the piezooptic ones on the basis of cumbersome relations including elastic compliances $\left(S_{v \mu}\right)$ give rise again to high final errors for the piezooptic parameters.

The piezooptic coefficients are mainly studied with interferometric or polarimetric techniques, which are sensitive enough to satisfy the required experimental accuracies. Nevertheless, the final results include large errors caused by a spatial stress distribution inside samples. Moreover, as we show below, this stress distribution (the corresponding stress values can deviate by $\sim 30 \%$ over the sample volume) exists even in the cases when the sample is loaded by homogeneous mechanical stress and the optical quality of material under study is high enough. As a result, the effective stress value and so the piezooptic coefficients are determined with insufficiently low accuracy.

One of the possible ways for solving the problem is creating stress distribution geometry in the sample, which is defined in advance. As an example (see, e.g., [15]), fourpoint bending method provides high accuracy of piezooptic polarimetric experiments, with the error not exceeding few per cents. However, the method requires a special (platelike) geometrical shape of samples and, moreover, many such plates are needed for complete piezooptic characterization of low-symmetry crystals.

On the other hand, the polarimetric studies of the piezooptic effect yield only in differences of the piezooptic coefficients, rather than separate coefficients of the piezooptic tensor themselves. In particular, the optical glasses are characterized by the stress-optic coefficient, which is in fact equal to the difference of coefficients of the piezooptic tensor. In their turn, the separate components of the piezooptic tensor can be obtained with utilizing interferometric technique, and then the knowledge of stress distribution inside the sample after its loading by uniaxial stress becomes important again.

In case of inhomogeneity of the stresses that appear inside the sample under uniaxial loading, the problem of accurate determination of piezooptic coefficients transforms into that of determination of stresses distribution inside the sample, i.e. the problem of stress tensor field tomography. The topics related to the stress tensor field tomography have been considered by many authors (see, e.g., [16-19]). It is known that the problem could be completely solved for some special cases assuming a priori information on the stress distribution [16]. Such a priori information is actually available in our case of uniaxial loading of sample, which permits employing polarimetric data only for a single projection as shown below.

In the present work we reveal the reasons for appearance of stress inhomogenity in the course of typical piezooptic experiments and demonstrate how to eliminate high corresponding errors or how these errors could properly be taken into account.

\section{Experimental procedure and mechanical model 2.1.Experimental procedure}

We studied the distribution of optical retardation in a glass sample using the imaging polarimeter described earlier in the work [20]. The functional scheme of the corresponding set-up is presented in Fig. 1.

Ukr. J. Phys. Opt. 2009, V10, №1 


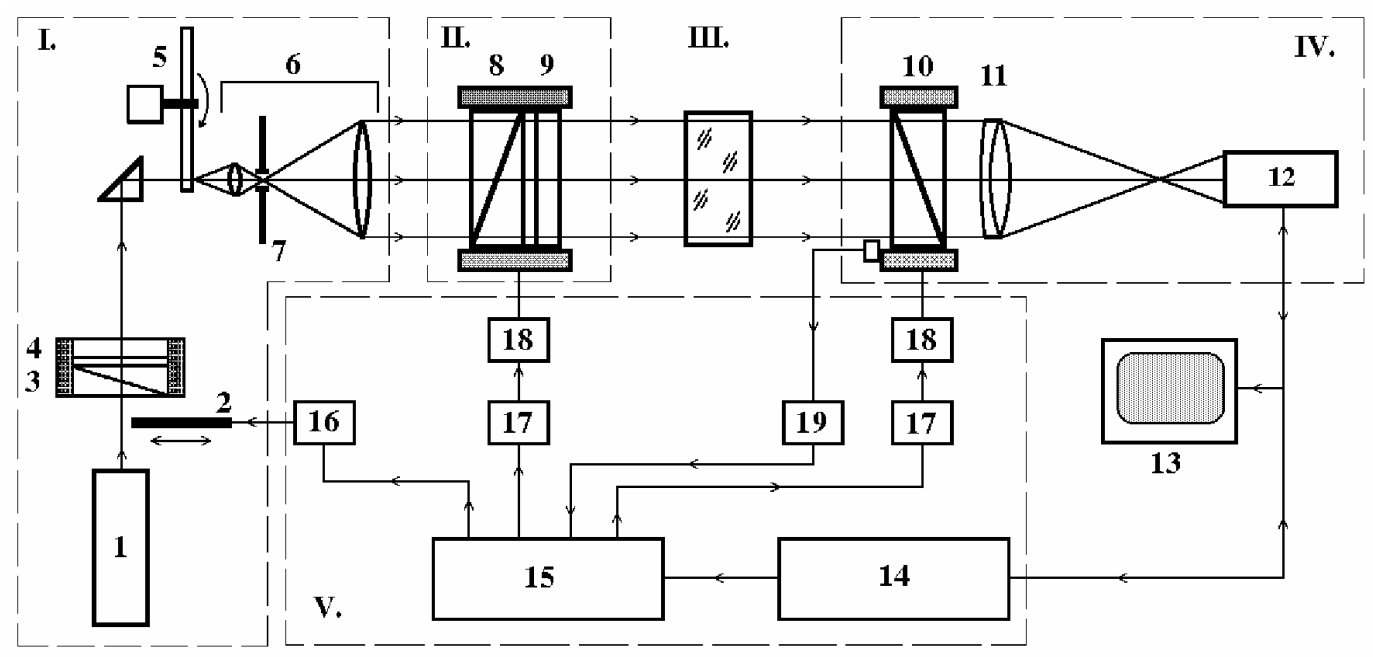

Fig. 1. Functional scheme of imaging polarimeter.

(I) Light generation section: 1 - laser; 2 - light shutter; 3 - polarizer, 4 - quarter-wave plate; 5 - coherence scrambler; 6 - beam expander; 7 - spatial filter;

(II) Polarization generation section: 8 - polarizer; 9 - quarter-wave plate; 10 - analyzer;

(III) Sample section (see Fig. 2);

(IV) Section of image analysis: 11 - objective lens; 12 - CCD camera;

(V) Operating section: 13 - monitor; 14 - camera interface; 15 - PC; 16 - shutter controller; 17 - step motor controller; 18 - step motor; 19 - position sensor controller.

The measuring procedure is as follow. A quarter-wave retarder (position 9 in Fig. 1) is aligned in $45^{\circ}$-position with respect to the polarizer (position 8 ). As a result, the light propagating from a polarization generator is circularly polarized. In order to measure polarization state of the light transmitted through the sample, the analyzer is rotated from $0^{\circ}$ to $180^{\circ}$. The sample image is recorded with the steps of $4.5 \mathrm{deg}$. Once the analyzer has reached the angle value of $90^{\circ}$, the light beam is shut out and the background image is recorded. The overall time of the measuring procedure is less than 30 seconds.

In case when the probing light is circularly polarized and the sample is well described by the model of linear optical retarder, the dependence of the light intensity $I$ on the analyzer azimuth $\alpha$ is expressed by the formula

$$
I=\frac{I_{0}}{2}\{1+\sin \Gamma \sin [2(\alpha-\varphi)]\},
$$

where $\varphi$ is the orientation angle of the optical indicatrix, $\Gamma=2 \pi \Delta n d / \lambda$ the optical retardation, $\lambda$ the light wavelength, $d$ the sample thickness and $\Delta n$ the optical birefringence. After recording and filtering of images, azimuthal dependences of the intensity $I$ are fitted by the sine function for each pixel of the image:

$$
I=C_{1}+C_{2} \cdot \sin \left[2\left(\alpha-C_{3}\right)\right]
$$

where $C_{1}, C_{2}$ and $C_{3}$ are fitting coefficients. 
Using the known relation for the optical retardation and the relation (3), one can write the fitting coefficients in the following form:

$$
C_{1}=\frac{I_{0}}{2}, \quad C_{2}=\frac{I_{0}}{2} \sin (\Gamma), \quad C_{3}=\varphi .
$$

It is seen that the optical retardation $\Gamma$ is determined by the fitting coefficients $C_{1}$ and $C_{2}$,

$$
\sin \Gamma=\frac{C_{2}}{C_{1}},
$$

while the angular orientation of the intensity minimum is determined by the orientation of principal axis $\varphi$ of the optical indicatrix and the coefficient $C_{3}$.Thus, after fitting the light intensity for each pixel of the sample image behind the analyzer as a function of polarization azimuth, one can construct 2D maps of the optical anisotropy parameters of the sample under test, namely the optical retardation and the orientation of the principal axis of its optical indicatrix.

A schematic view of the loading device used in our experiment is shown in Fig. 2. Between the sample and the top and bottom dies, the cardboards are used as intermediate layers, in order to eliminate inhomogeneity of the corresponding surfaces and decrease the friction force between these surfaces. The sample in the shape of cube with the dimensions $(\mathrm{X}) 11.45 \times(\mathrm{Y}) 11.3 \times(\mathrm{Z}) 11.45 \mathrm{~mm}^{3}$ was prepared from BK7 (Schott classification) glass. Its refractive index for the wavelength of $\lambda=632.8 \mathrm{~nm}$ is equal to

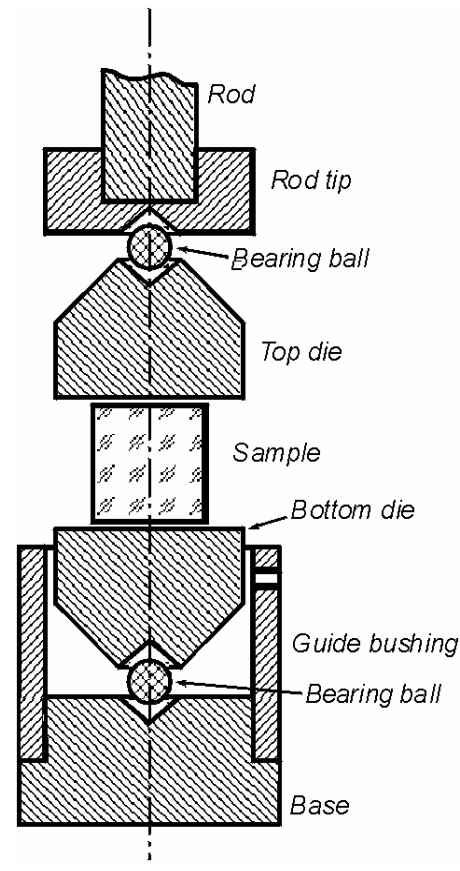

Fig. 2. Scheme of the loading device. $n=1.51466$, while the difference of the piezooptic coefficients amounts to $\frac{1}{2} n_{0}^{3}\left(\pi_{11}-\pi_{12}\right)=2.76 \times 10^{-12} \mathrm{~m}^{2} / \mathrm{N}$ at $\lambda=550 \mathrm{~nm}$ [21] (in the present study we neglect the dispersion of piezooptic coefficients in the spectral region of $550-632.8 \mathrm{~nm}$ ).

\subsection{Mechanical model and simulations}

Let us assume that the experimental conditions provide a possibility for uniform application of mechanical load in the $Z$ direction of sample and that the sample is initially optically homogeneous and isotropic. The optical radiation propagates along the $Y$ direction. Then inhomogeneity of the mechanical stresses inside the sample can appear only due to a friction force between the upper and lower sample surfaces and the intermediate card- 
board layers. The maximum value of this friction forces $d F_{\max }$ are proportional to the loading stress $\sigma_{3}$,

$$
d F_{\max }=k \sigma_{3} d S
$$

where $k=0.22$ is the friction coefficient for the case of friction between the glass and the paper [22] and $d S$ denotes the small element of the square of the surface of the loaded sample. These friction forces are directed from the lateral faces of the sample to the central $Z$ axis (Fig. 3), thus leading to appearance of barrel-shaped distortion of the sample under the compressive stress $\sigma_{3}$.

In the first approximation we take into

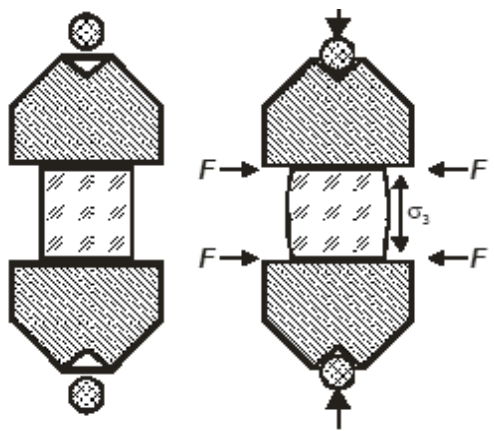

Fig. 3. Barrel-shaped distortion of the sample under compressive stress $\sigma_{3}$. account the following boundary conditions for this type of inhomogeneously stressed sample:

(1) $\sigma_{1}=\sigma_{2}=0$ on four lateral faces of the sample;

(2) $\sigma_{1}^{\max }=\sigma_{2}^{\max }=k \sigma_{3}$ on the upper and lower faces;

(3) the value $\sigma_{3}$ remains the same on all the faces (in our experiment and simulations we have $\sigma_{3}=-1.93 \times 10^{6} \mathrm{~N} / \mathrm{m}^{2}$ ).

Let us use the approach presented in the work [23] for the superposition of three solutions, each of which is a solution of the problem for elastic layer. The general solution of the Lame's equation under the condition of absence of the volume forces can be written as a sum of three solutions for the displacement vectors $u, v, w$ along $X, Y$ and $Z$ directions, respectively [23]:

$$
\begin{aligned}
& u=A_{0} \frac{\partial^{2} F_{x}^{0}}{\partial x^{2}}+\nabla^{2} F_{x}^{0}+A_{0} \frac{\partial^{2} F_{y}^{0}}{\partial x \partial y}-\frac{\partial f_{y}}{\partial z}+A_{0} \frac{\partial^{2} F_{z}^{0}}{\partial x \partial z}+\frac{\partial f_{z}}{\partial y}, \\
& v=A_{0} \frac{\partial^{2} F_{x}^{0}}{\partial x \partial y}+\frac{\partial f_{x}}{\partial z}+A_{0} \frac{\partial^{2} F_{y}^{0}}{\partial y^{2}}+\nabla^{2} F_{y}^{0}+A_{0} \frac{\partial^{2} F_{z}^{0}}{\partial y \partial z}-\frac{\partial f_{z}}{\partial x}, \\
& w=A_{0} \frac{\partial^{2} F_{x}^{0}}{\partial x \partial z}-\frac{\partial f_{x}}{\partial y}+A_{0} \frac{\partial^{2} F_{y}^{0}}{\partial y \partial z}+\frac{\partial f_{y}}{\partial x}+A_{0} \frac{\partial^{2} F_{z}^{0}}{\partial z^{2}}+\nabla^{2} F_{z}^{0},
\end{aligned}
$$

where $\nabla^{2} \nabla^{2} F_{x, y, z}^{0}=0, \nabla^{2} f_{x, y, z}=0, A_{0}=-\frac{\lambda+\mu}{\lambda+2 \mu}$, and $\lambda$ and $\mu$ are the Lame's coefficients. The position of our sample is determined by $0 \leq x \leq a, 0 \leq y \leq b$ and $0 \leq z \leq c$ 
( $a, b, c$ being the lengths of the sample along $X, Y$ and $Z$ directions, respectively). The functions $F_{x, y, z}^{0}$ and $f_{x, y, z}$ may be written as follows:

$$
\begin{aligned}
F_{x}^{0}= & \sum_{n=1}^{\infty} \sum_{m=1}^{\infty}\left\{\left[K_{1}^{n m} \sinh \left(x r_{n m}\right)+K_{2}^{n m} \cosh \left(x r_{n m}\right)+K_{3}^{n m} x \sinh \left(x r_{n m}\right)+\right.\right. \\
& \left.\left.+K_{4}^{n m} x \cosh \left(x r_{n m}\right)\right] \sin \frac{n \pi y}{b} \sin \frac{m \pi z}{c}\right\}, r_{n m}=\pi \sqrt{\frac{n^{2}}{b^{2}}+\frac{m^{2}}{c^{2}}} \\
F_{y}^{0}= & \sum_{k=1}^{\infty} \sum_{m=1}^{\infty}\left\{\left[L_{1}^{k m} \sinh \left(y r_{k m}\right)+L_{2}^{k m} \cosh \left(y r_{k m}\right)+L_{3}^{k m} y \sinh \left(y r_{k m}\right)+\right.\right. \\
& \left.\left.+L_{4}^{k m} y \cosh \left(y r_{k m}\right)\right] \sin \frac{k \pi x}{a} \sin \frac{m \pi z}{c}\right\}, \quad r_{k m}=\pi \sqrt{\frac{k^{2}}{a^{2}}+\frac{m^{2}}{c^{2}}} \\
F_{z}^{0}= & \sum_{k=1}^{\infty} \sum_{n=1}^{\infty}\left\{\left[M_{1}^{k n} \sinh \left(z r_{k n}\right)+M_{2}^{k n} \cosh \left(z r_{k n}\right)+M_{3}^{k n} z \sinh \left(z r_{k n}\right)+\right.\right. \\
& \left.\left.+M_{4}^{k n} z \cosh \left(z r_{k n}\right)\right] \sin \frac{k \pi x}{a} \sin \frac{n \pi y}{b}\right\}, \quad r_{k m}=\pi \sqrt{\frac{k^{2}}{a^{2}}+\frac{n^{2}}{b^{2}}}, \\
f_{x}= & \sum_{n=0}^{\infty} \sum_{m=0}^{\infty}\left\{\left[K_{5}^{n m} \sinh \left(x r_{n m}\right)+K_{6}^{n m} \cosh \left(x r_{n m}\right)\right] \cos \frac{n \pi y}{b} \cos \frac{m \pi z}{c}\right\}, \\
f_{y}= & \sum_{k=0}^{\infty} \sum_{m=0}^{\infty}\left\{\left[L_{5}^{k m} \sinh \left(y r_{k m}\right)+L_{6}^{k m} \cosh \left(y r_{k m}\right)\right] \cos \frac{k \pi x}{a} \cos \frac{m \pi z}{c}\right\}, \\
f_{z}= & \sum_{k=0}^{\infty} \sum_{n=0}^{\infty}\left\{\left[M_{5}^{k n} \sinh \left(z r_{k n}\right)+M_{6}^{k n} \cosh \left(z r_{k n}\right)\right] \cos \frac{k \pi x}{a} \cos \frac{m \pi y}{b}\right\} .
\end{aligned}
$$

Inserting equations (8)-(11) into (7) gives

$$
\begin{aligned}
u & =\sum_{n=1}^{\infty} \sum_{m=1}^{\infty} \Theta_{1}^{n m}(x) \sin \frac{n \pi y}{b} \sin \frac{m \pi z}{c}+\sum_{k=0}^{\infty} \sum_{m=1}^{\infty} \Theta_{2}^{k m}(y) \cos \frac{k \pi x}{a} \cos \frac{m \pi z}{c}+ \\
& +\sum_{k=0}^{\infty} \sum_{n=1}^{\infty} \Theta_{3}^{k n}(z) \cos \frac{k \pi x}{a} \sin \frac{n \pi y}{b}, \\
v & =\sum_{n=0}^{\infty} \sum_{m=1}^{\infty} \Theta_{4}^{n m}(x) \cos \frac{n \pi y}{b} \sin \frac{m \pi z}{c}+\sum_{k=1}^{\infty} \sum_{m=1}^{\infty} \Theta_{5}^{k m}(y) \sin \frac{k \pi x}{a} \sin \frac{m \pi z}{c}+ \\
& +\sum_{k=1}^{\infty} \sum_{n=0}^{\infty} \Theta_{6}^{k n}(z) \sin \frac{k \pi x}{a} \cos \frac{n \pi y}{b}, \\
w & =\sum_{n=1}^{\infty} \sum_{m=0}^{\infty} \Theta_{7}^{n m}(x) \sin \frac{n \pi y}{b} \cos \frac{m \pi z}{c}+\sum_{k=1}^{\infty} \sum_{m=0}^{\infty} \Theta_{8}^{k m}(y) \sin \frac{k \pi x}{a} \cos \frac{m \pi z}{c}+ \\
& +\sum_{k=1}^{\infty} \sum_{n=1}^{\infty} \Theta_{9}^{k n}(z) \sin \frac{k \pi x}{a} \sin \frac{n \pi y}{b} .
\end{aligned}
$$


Relations (12) should be completed by the equations that follow from the Hooke's law,

$$
\begin{array}{ll}
\sigma_{1}=2 \mu \frac{\partial u}{\partial x}+\lambda\left(\frac{\partial u}{\partial x}+\frac{\partial v}{\partial y}+\frac{\partial w}{\partial z}\right), & \sigma_{4}=\mu\left(\frac{\partial w}{\partial y}+\frac{\partial v}{\partial z}\right), \\
\sigma_{2}=2 \mu \frac{\partial v}{\partial y}+\lambda\left(\frac{\partial u}{\partial x}+\frac{\partial v}{\partial y}+\frac{\partial w}{\partial z}\right), & \sigma_{5}=\mu\left(\frac{\partial w}{\partial x}+\frac{\partial u}{\partial z}\right), \\
\sigma_{3}=2 \mu \frac{\partial w}{\partial z}+\lambda\left(\frac{\partial u}{\partial x}+\frac{\partial v}{\partial y}+\frac{\partial w}{\partial z}\right), & \sigma_{6}=\mu\left(\frac{\partial u}{\partial y}+\frac{\partial v}{\partial x}\right) .
\end{array}
$$

The functions $\Theta_{1}^{n m}(x), \Theta_{2}^{k m}(y), \Theta_{3}^{k n}(z), \Theta_{4}^{n m}(x), \Theta_{5}^{k m}(y), \Theta_{6}^{k n}(z), \Theta_{7}^{n m}(x), \Theta_{8}^{k m}(y)$, $\Theta_{9}^{k n}(z)$ in (12) depend on the coordinates, the number of harmonics and the coefficients $K_{i}^{n m}, L_{i}^{k m}, M_{i}^{k n}(i=1, \ldots 6$ - see relations (8)-(11)). These coefficients are determined after solving the set of equations (13) and accounting for the boundary conditions for the sample faces mentioned above. With the known coefficients and the relations (13), the stress distribution can be determined for all the sample volume.

Simulations of distribution of the optical retardation have been performed on the basis of integral Jones matrix approach. The sample under test has been divided into one thousand $(10 \times 10 \times 10)$ elementary, optically uniform cells. The resulting Jones matrices for each of one hundred elementary beams $(i, j=1, \ldots 10)$ have been obtained by multiplying the Jones matrices of ten $(n)$ elementary cells, trough which each elementary beam has passed:

$$
J^{i j}=\prod_{n=1}^{10} J_{n}^{i j}
$$

where

$$
\begin{aligned}
& J_{n}^{i j}=\left|\begin{array}{cc}
\left.e^{i \Gamma_{n}^{i j} / 2} \cos ^{2} \xi_{n}^{i j}+e^{-i \Gamma_{n}^{i j} / 2} \sin ^{2} \xi_{n}^{i j}\right) & i \sin \left(\Gamma_{n}^{i j} / 2\right) \sin 2 \xi_{n}^{i j} \\
i \sin \left(\Gamma_{n}^{i j} / 2\right) \sin 2 \xi_{n}^{i j} & \left(e^{i \Gamma_{n}^{i j} / 2} \sin ^{2} \xi_{n}^{i j}+e^{-i \Gamma_{n}^{i j} / 2} \cos ^{2} \xi_{n}^{i j}\right)
\end{array}\right|, \\
& \Gamma_{n}^{i j}=\frac{2 \pi d_{n}^{i j}}{\lambda}\left\{\frac{1}{2} n_{0}^{3}\left(\pi_{11}-\pi_{12}\right)\left(\left(\sigma_{1}\right)_{n}^{i j}-\left(\sigma_{3}\right)_{n}^{i j}\right) \sqrt{1+\left(\frac{2\left(\sigma_{5}\right)_{n}^{i j}}{\left(\sigma_{1}\right)_{n}^{i j}-\left(\sigma_{3}\right)_{n}^{i j}}\right)^{2}}\right\}, \\
& \xi_{n}^{i j}=\frac{1}{2} \arctan \frac{2\left(\sigma_{5}\right)_{n}^{i j}}{\left(\sigma_{1}\right)_{n}^{i j}-\left(\sigma_{3}\right)_{n}^{i j}}
\end{aligned}
$$

Since $\left|\begin{array}{l}E_{1}^{i j} \\ E_{3}^{i j}\end{array}\right|=J^{i j}\left|\begin{array}{c}E_{1} \\ E_{3}\end{array}\right|, \quad E_{1}=1, \quad E_{3}=i$, the resulting optical retardation for the each elementary beam is determined by the relation

$$
\Gamma^{i j}=\arctan \left\{\operatorname{Im}\left(E_{1}^{i j} / E_{3}^{i j}\right) / \operatorname{Re}\left(E_{1}^{i j} / E_{3}^{i j}\right)\right\} .
$$




\section{Experimental results}

In Fig. 4 we represent distribution of the optical retardation and the orientation of principal axis of the optical indicatrix for the optical tract including no sample, as a demonstration of accuracy of our experiment. Of course, the optical retardation in the optical tract which is filled only with air and isotropic optical elements such as lenses and polarizers should be equal to zero. However, some false "background" retardation still exists due to experimental errors caused mainly by multiple light reflections in the optical elements and small misalignments of optical axes of those elements, which are being rotated in the course of experimental procedures. Thus, following from the results presented in Fig. 4, one can determine the apparatus errors for evaluation of the optical retardation and the orientation of the optical indicatrix as $\pm 2 \mathrm{deg}$ and $\pm 5 \mathrm{deg}$, respectively.

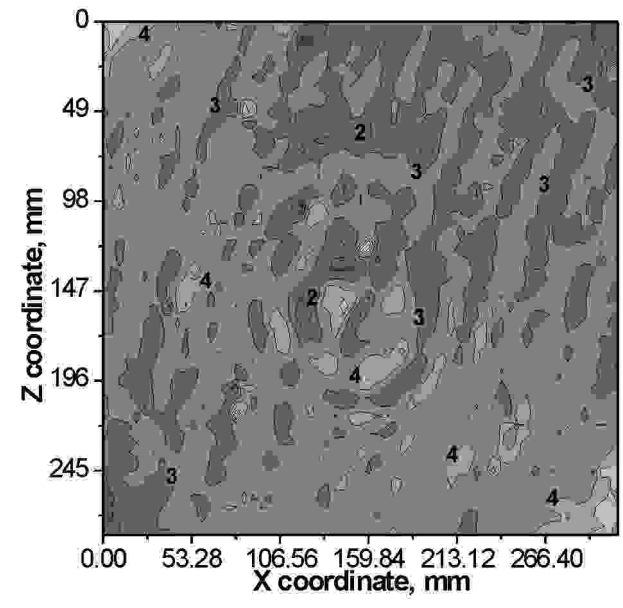

(a)

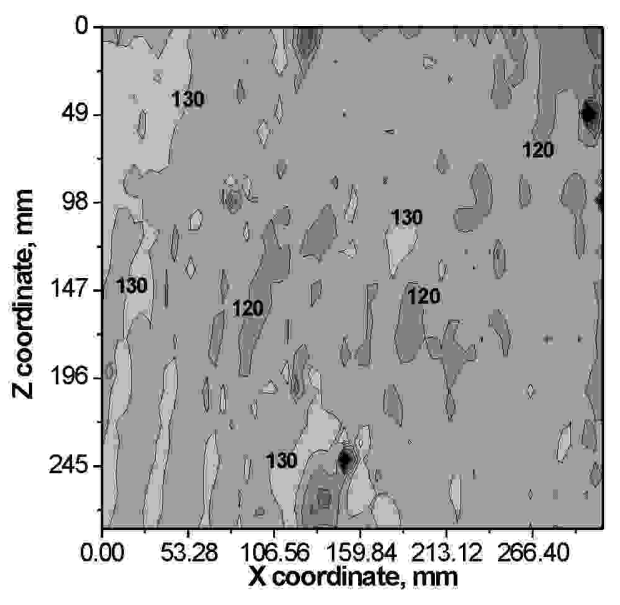

(b)

Fig. 4. Images of distribution of the optical retardation (a) and the orientation of principal axis of the optical indicatrix (b) (in deg) for the optical tract in our set-up including no sample.

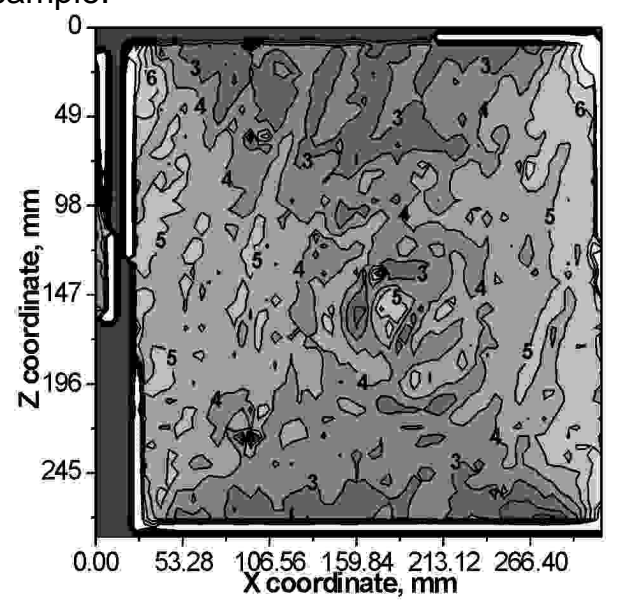

(a)

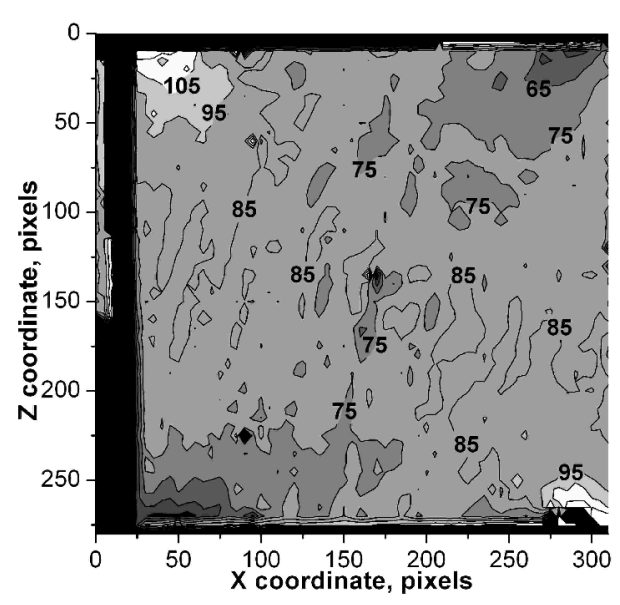

(b)

Fig. 5. Images of distributions of the optical retardation (a) and the orientation of principal axis of the optical indicatrix (b) (in deg) for the glass sample with no mechanical stress applied.

Ukr. J. Phys. Opt. 2009, V10, №1 
The distributions of the optical retardation and the orientation of principal axis of the optical indicatrix for the glass sample with no mechanical stress applied are presented in Fig. 5. From those results it follows that the residual optical birefringence is smaller than $6 \times 10^{-7}$. Moreover, one can see from comparison of the maps presented in Fig. 4 and Fig. 5 that the major part of this birefringence is false, being caused by peculiarities of both our experimental set-up and the method used, i.e. it is actually an apparatus error. In fact, the birefringence as small as $6 \times 10^{-7}$ merely represents the accuracy for the birefringence achieved in the present experiment.

The distributions of the optical retardation and the orientation of principal axis of the optical indicatrix for the glass sample in the conditions of the mechanical stress $\sigma_{3}=-1.93 \times 10^{6} \mathrm{~N} / \mathrm{m}^{2}$ applied are displayed in Fig. 6. It is important that repeated experiments accompanied with realignments of the sample have not led to notable difference in the distributions of the optical parameters (the optical phase differences throughout the cross section of the sample and the cross section of circular-section laser beam with the diameter $1.5 \mathrm{~mm}$ propagated through the centre of the cross section are compared in Table 1). However, the maximum of the optical retardation has been positioned at different points, i.e. in different experiments it has been close to lateral, upper or bottom edges of the sample. These shifts are probably caused by some misalignments of stress application, leading to appearance of additional components of the stress tensor.

It is seen that the relative error of determination of optical retardation for the whole cross section of the sample approaches $14 \%$, while the relative error for the cross section of circular-section laser beam is about $4 \%$. In other words, the error for the experiments with non-expanded laser beam can be reduced at least by 3.5 times, provided that we know the value of mechanical stress in the spatial region where laser beam propagates.

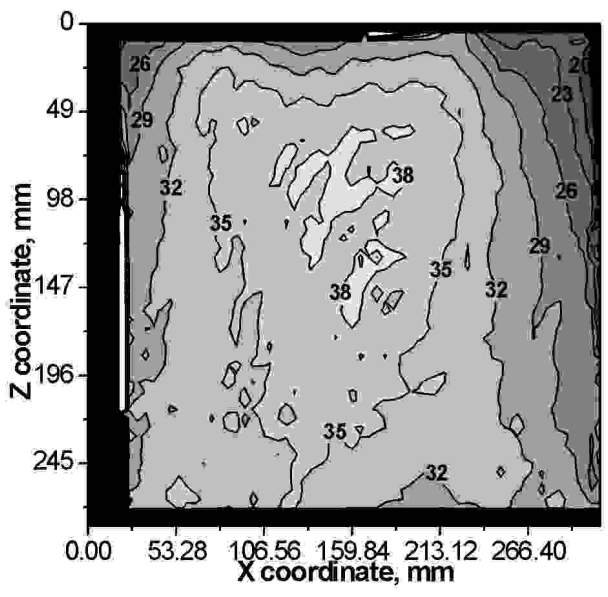

(a)

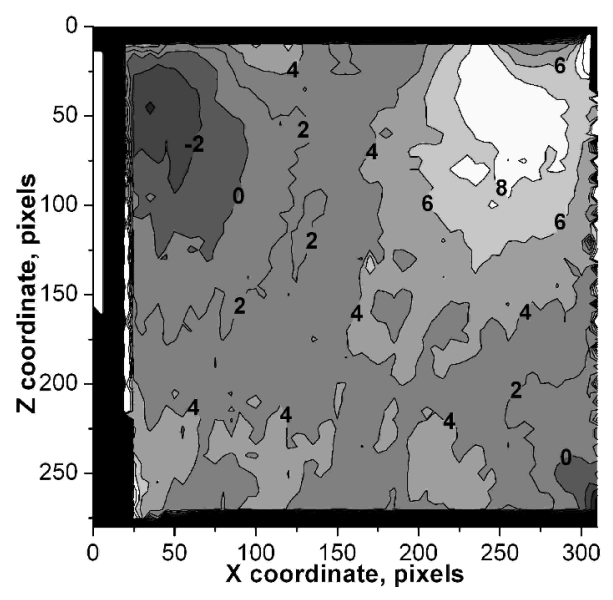

(b)

Fig. 6. Images of distributions of the optical retardation (a) and the orientation of principal axis of the optical indicatrix (b) (in deg) for the glass sample under the mechanical stress $\sigma_{3}=-1.93 \times 10^{6} \mathrm{~N} / \mathrm{m}^{2}$ applied. 
Table 1. Optical retardation for the whole cross section of the sample and for the cross section of circular-section laser beam with the diameter of $1.5 \mathrm{~mm}$ propagated through the centre of the sample.

\begin{tabular}{|c|c|c|c|c|}
\hline $\begin{array}{c}\text { Number of } \\
\text { experiment }\end{array}$ & $\begin{array}{c}\text { Retardation for the } \\
\text { whole cross section } \\
\text { of sample, deg }\end{array}$ & $\begin{array}{c}\text { Relative } \\
\text { error, } \%\end{array}$ & $\begin{array}{c}\text { Retardation for the cross } \\
\text { section of circular- } \\
\text { section laser beam, deg }\end{array}$ & $\begin{array}{c}\text { Relative } \\
\text { error, \% }\end{array}$ \\
\hline 1 & $33.06 \pm 4.85$ & 14.66 & $37.58 \pm 0.97$ & 2.59 \\
\hline 2 & $33.28 \pm 5.19$ & 15.58 & $38.68 \pm 1.81$ & 4.67 \\
\hline 3 & $31.70 \pm 4.40$ & 13.87 & $35.89 \pm 1.62$ & 4.50 \\
\hline 4 & $31.71 \pm 5.41$ & 17.07 & $34.89 \pm 1.44$ & 4.13 \\
\hline 5 & $31.70 \pm 3.26$ & 10.30 & $34.32 \pm 1.27$ & 3.71 \\
\hline 6 & $32.19 \pm 3.89$ & 12.09 & $35.52 \pm 1.58$ & 4.46 \\
\hline Mean value & $32.27 \pm 4.50$ & 13.94 & $36.15 \pm 1.45$ & 4.01 \\
\hline
\end{tabular}

\section{Discussion}

In Fig. 7 we present the simulated optical retardation for the glass sample loaded by mechanical stress component $\sigma_{3}$. In these simulations we have employed the boundary conditions mentioned above: $\sigma_{1}=\sigma_{2}=0$ on all of the four lateral faces of the sample; $\sigma_{1}=\sigma_{2}=k \sigma_{3}$ on the upper and lower faces; $\sigma_{3}$ has the same value on all the faces ( $\sigma_{3}=-1.93 \times 10^{6} \mathrm{~N} / \mathrm{m}^{2}$ in our experiment and simulations). We have used relation (17) and 161 harmonics in the expansion (12).

As one can see from Fig. 7b, the major part of the cross section (75\%) satisfies the boundary conditions suggested by us, with the accuracy of \pm 3 deg for the optical retardation. When comparing Fig. 6a and Fig. 7a, one can see that the maximum of optical retardation obtained experimentally is shifted towards the upper edge of the sample. This shift

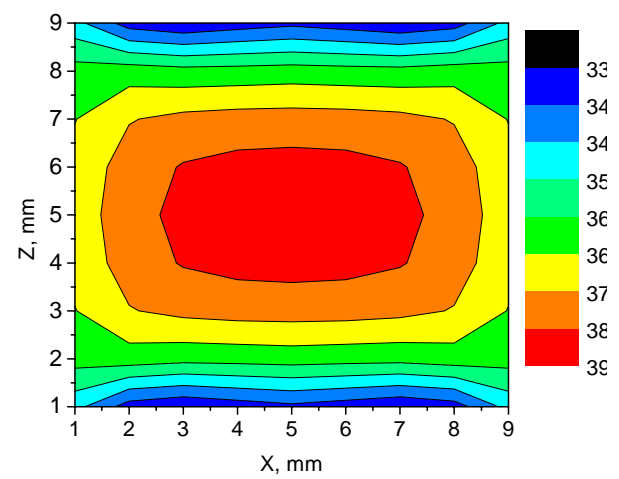

(a)

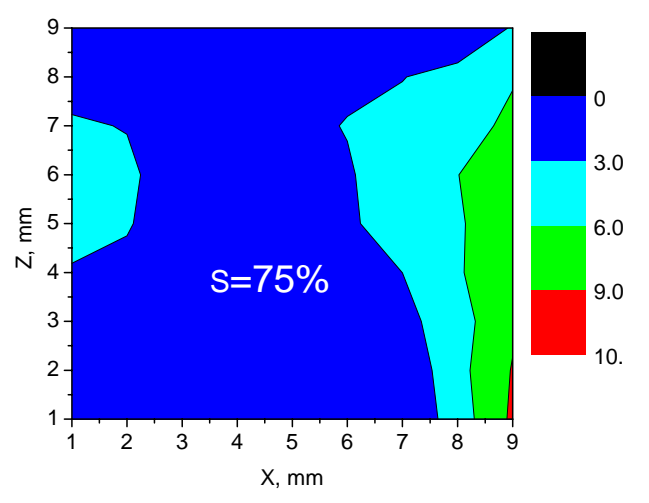

(b)

Fig. 7. Maps of the simulated optical retardation (a) and the difference of experimental (see Fig. 6a) and simulated optical retardations (b) (in deg). 
can be produced by a slight inhomogeneity of the stress applied to the upper surface of the sample. For example, if the stress is applied to the upper surface of the sample with some misalignment, this stress is probably deviated from the initial flat profile to a sinusoidal (half-period) profile. In such a case the stress distribution on the upper face of the sample can be described by the relation

$$
\sigma_{3}=-N_{1}\left[0.5+N_{2} \sin \pi \frac{x}{11.45} \sin \pi \frac{y}{11.3}\right],
$$

where $N_{1}$ and $N_{2}$ are the normalization factors which should satisfy the condition $\iint_{c} \sigma_{3} d x d y=-249.7 \mathrm{~N}$ (the magnitude $-249.7 \mathrm{~N}$ corresponds to the force applied). In the simulations we have used the expansions (8)-(11) with $K_{i}^{m n}(i=1 \ldots 6, m, n=1 \ldots 31), L_{i}^{k m}$ $(i=1 \ldots 6, k, m=1 \ldots 31)$ and $M_{i}^{k n}(i=1 \ldots 6, k, n=1 \ldots 9)$.

The results of the simulations are presented in Fig. 8. Fig. 8a testifies that the maximum of optical retardation on the simulated map is shifted towards the upper edge of the sample. This satisfies qualitatively better the optical retardation map obtained experimen-

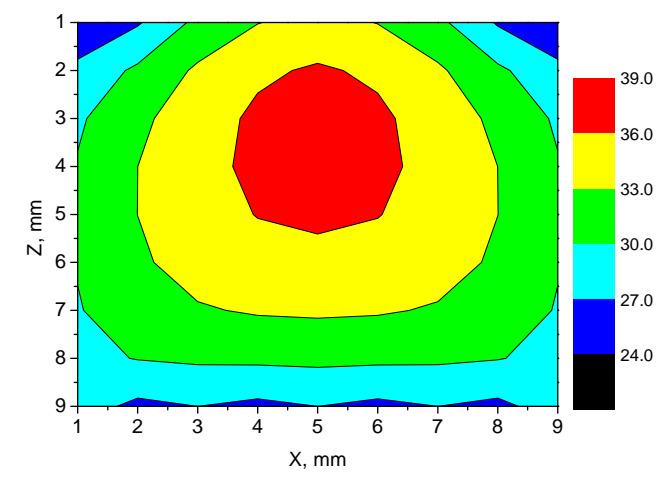

(a)

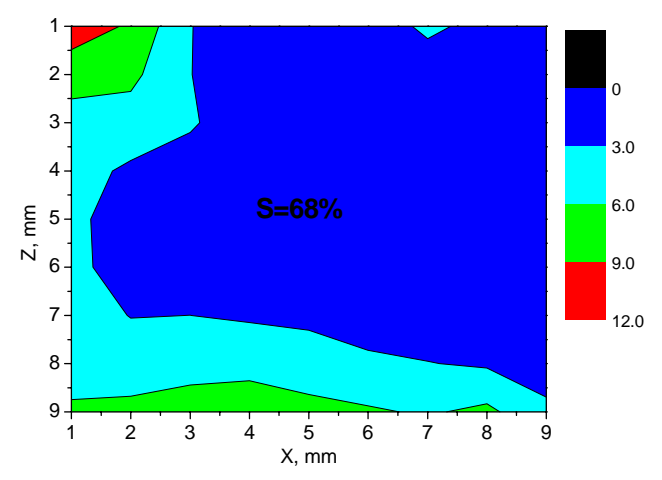

(b)

Fig. 8. Maps of the simulated optical retardation (a) and the difference of experimental (see Fig. 6a) and simulated optical retardations (b) (in deg), with additional consideration of sinusoidal distribution of mechanical stress on the upper sample surface.

tally. However, in this case a smaller part of the cross section (68\%) agrees with the experimental results. The corresponding misalignment of experimentally obtained and simulated maps may be caused by the two following reasons. The first one consists in rather approximate character of the boundary condition $\sigma_{1}=\sigma_{2}=0$ on the lateral faces of the sample. Indeed, if a barrel-shaped distortion of the sample takes place, the distribution of $\sigma_{1}$ and $\sigma_{2}$ on the lateral faces $Y=0, Y=b$ and $X=0, X=a$ can be described respectively by the relations

$$
\begin{aligned}
& \left.\sigma_{1}\right|_{x=0, a}=0,\left.\quad \sigma_{2}\right|_{x=0, a}=-0.2 \times \sigma_{3} \sin \pi \frac{z}{c} \sin \pi \frac{y}{b}, \\
& \left.\sigma_{2}\right|_{y=0, b}=0,\left.\quad \sigma_{1}\right|_{y=0, b}=-0.2 \times \sigma_{3} \sin \pi \frac{x}{a} \sin \pi \frac{z}{c},
\end{aligned}
$$


where -0.2 is the fitting parameter. The second reason is the fact that the maximum of optical retardation on the experimental map is shifted not only towards the upper face of the sample but also leftwards. This can be taken into account while modifying the relation (18):

$$
\begin{aligned}
& \left.\sigma_{3}\right|_{z=c}=-N_{1}\left[0.5+N_{2} \sin \pi \frac{x}{a} \sin \pi \frac{y}{b}+\frac{\pi^{2}}{48}(1-x / a)(1-y / b)\right], \\
& \left.\sigma_{3}\right|_{z=0}=-N_{1}\left[1+\frac{\pi^{2}}{48}(1-x / a)(1-y / b)+\frac{\pi}{8} x / a\right],
\end{aligned}
$$

where $N_{1}$ and $N_{2}$ are the normalization factors which should satisfy the conditions $\iint_{z=c} \sigma_{3} d x d y=-249.7 \mathrm{~N}$ and $\iint_{z=0} \sigma_{3} d x d y=-249.7 \mathrm{~N}$. The results of the corresponding simulations are shown in Fig. 9.

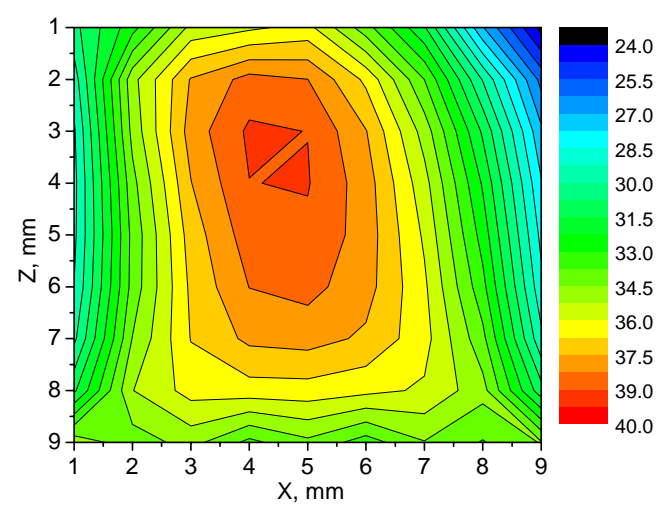

(a)

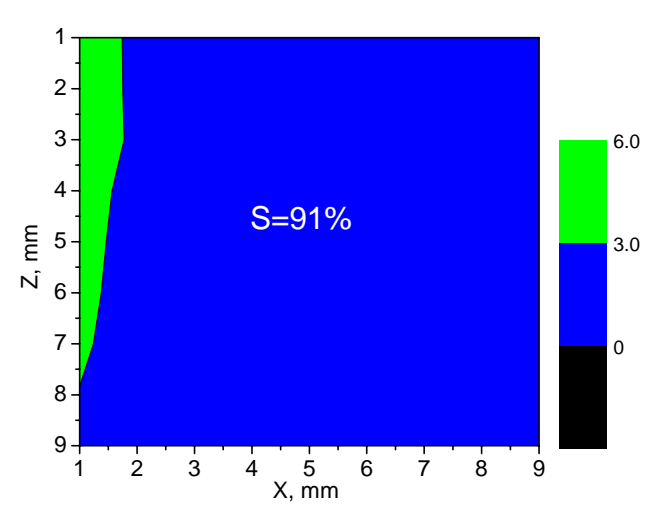

(b)

Fig. 9. Maps of the simulated optical retardation (a) and the difference of experimental (see Fig. 6a) and simulated optical retardations (b) (in deg), with additional consideration of conditions (19) and (20).

Figure $9 \mathrm{~b}$ shows that the major part of the cross section (91\%) becomes correlated with the experimental results. Nonetheless, some small part of the cross section (9\%) still disagrees with the experimental results. The disagreement is probably caused by some warp of the top die in the upper left side of the sample, along with some sliding between the sample and the dies. The last condition may be accounted for with reducing the friction coefficient down to the value $k=0.1$. In this case the boundary conditions should be supplemented by the relations

$$
\left.\sigma_{3}\right|_{z=c}=-N_{1}\left[-0.05 \sin \pi \frac{x}{a} \sin \pi \frac{y}{b}+0.3 \frac{\pi^{2}}{48}(1-x / a)(1-y / b)\right] .
$$

The results of the relevant simulations are shown in Fig. 10. As one can readily see, we have reached the situation when the experimental and simulation results have the similarity of $98 \%$. This enables one to finally reconstruct the stress tensor field inside the sample (see Fig. 11). 


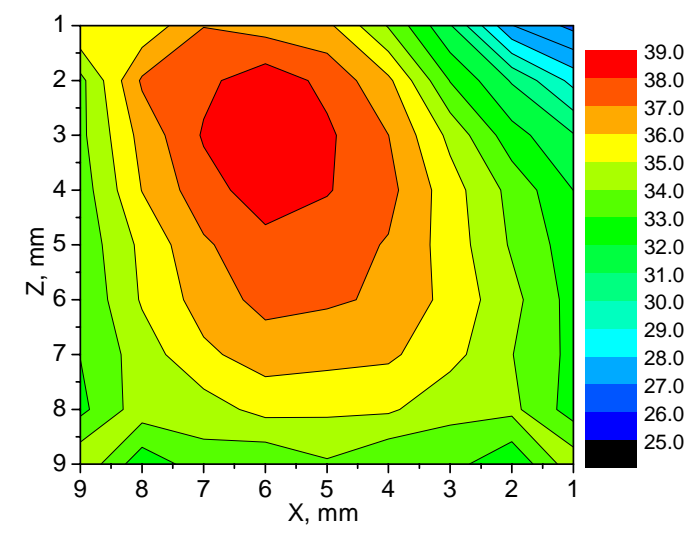

(a)

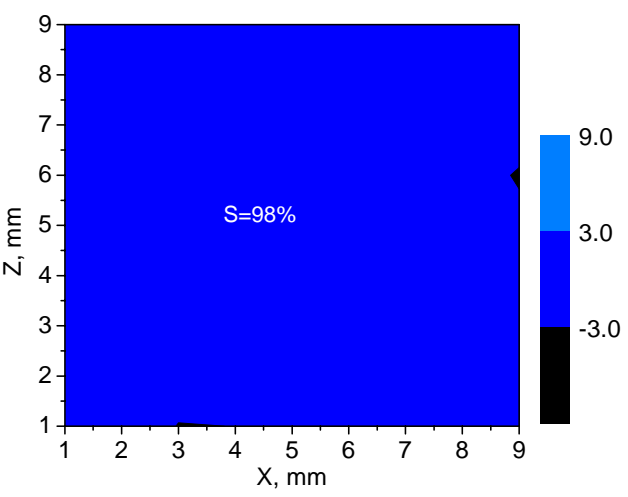

(b)

Fig. 10. Maps of the simulated optical retardation (a) and the difference of experimental (see Fig. 6a) and simulated optical retardations (b) (in deg), with consideration of additional condition (21) and taking into account of sliding between the sample and the dies.

Basing on the results presented in Fig. 11, we are in a position to draw the following conclusions:

- as a result of loading the sample in a manner described above, all the components of the stress tensor appear;

- the distribution of the mechanical stress tensor components inside the sample is inhomogeneous;

- the $\sigma_{3}$ component in the vicinity of geometrical centre of the sample is one order of magnitude larger than the $\sigma_{1}$ component and two orders of magnitude larger than the other stress components;

- the $\sigma_{3}$ component in the centre of the sample reaches a value $26 \%$ higher than the stress actually loaded, while the deviation of this component within the sample volume is equal to $32 \%$.

- the deviations of the other stress tensor components exceed hundred per cents and, moreover, the signs of the shift stress components are different in different parts of the sample.

Let us assume that a non-expanded laser beam with the cross section area $\sim 2 \mathrm{~mm}^{2}$ propagates through the sample centre parallel to the Y axis. It follows from Fig. 10 that the optical retardation for this light propagation direction is equal to $37.4 \mathrm{deg}$. On the other hand, the value of optical retardation for the case of homogeneously distributed mechanical stress $\left(\sigma_{3}=-1.93 \times 10^{6} \mathrm{~N} / \mathrm{m}^{2}\right)$ calculated with the formula

$$
\Gamma=\frac{\pi d}{\lambda} n_{0}^{3}\left(\pi_{11}-\pi_{12}\right) \sigma_{3}
$$

(with $n_{0}^{3}\left(\pi_{11}-\pi_{12}\right)=5.52 \times 10^{-12} \mathrm{~m}^{2} / \mathrm{N}$ ) is equal to $34.2 \mathrm{deg}$. Thus, the actual value of the optical retardation is smaller by $8.5 \%$ than the measured one. This means that in any prac 

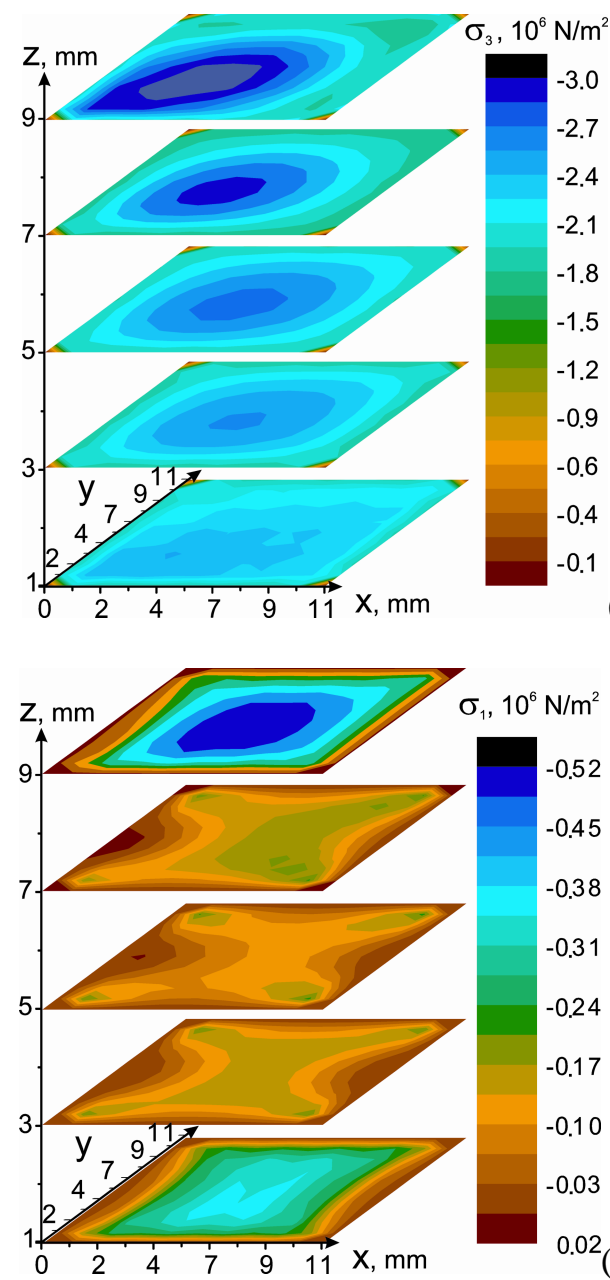

(a)
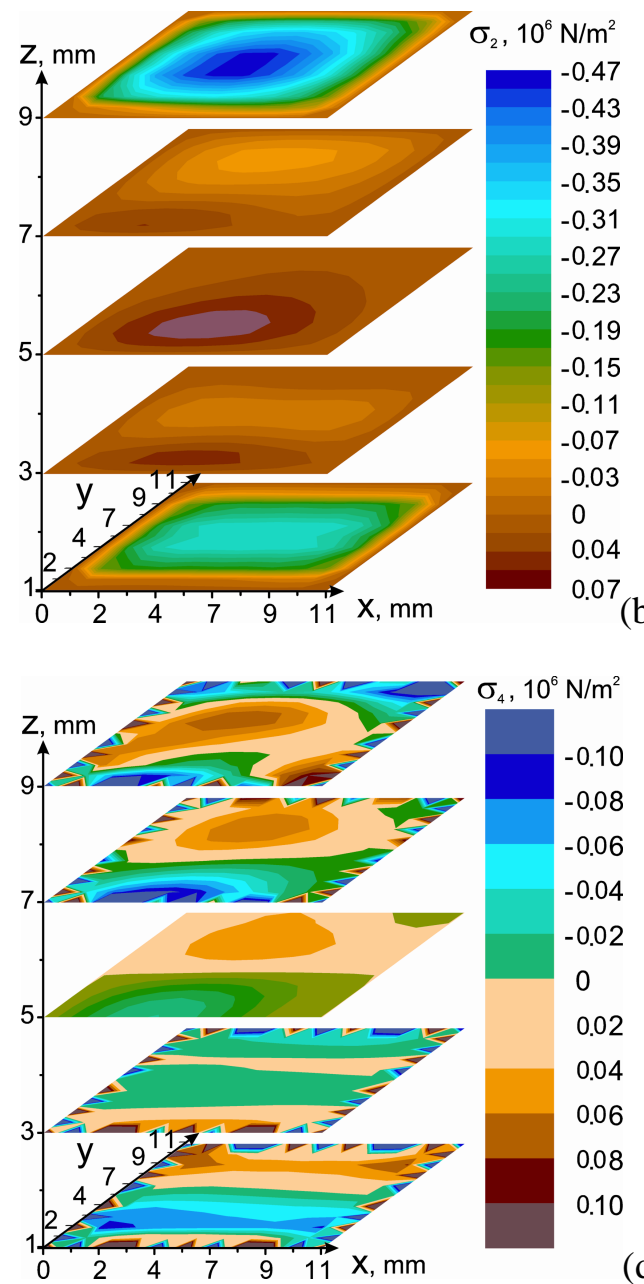

(d)
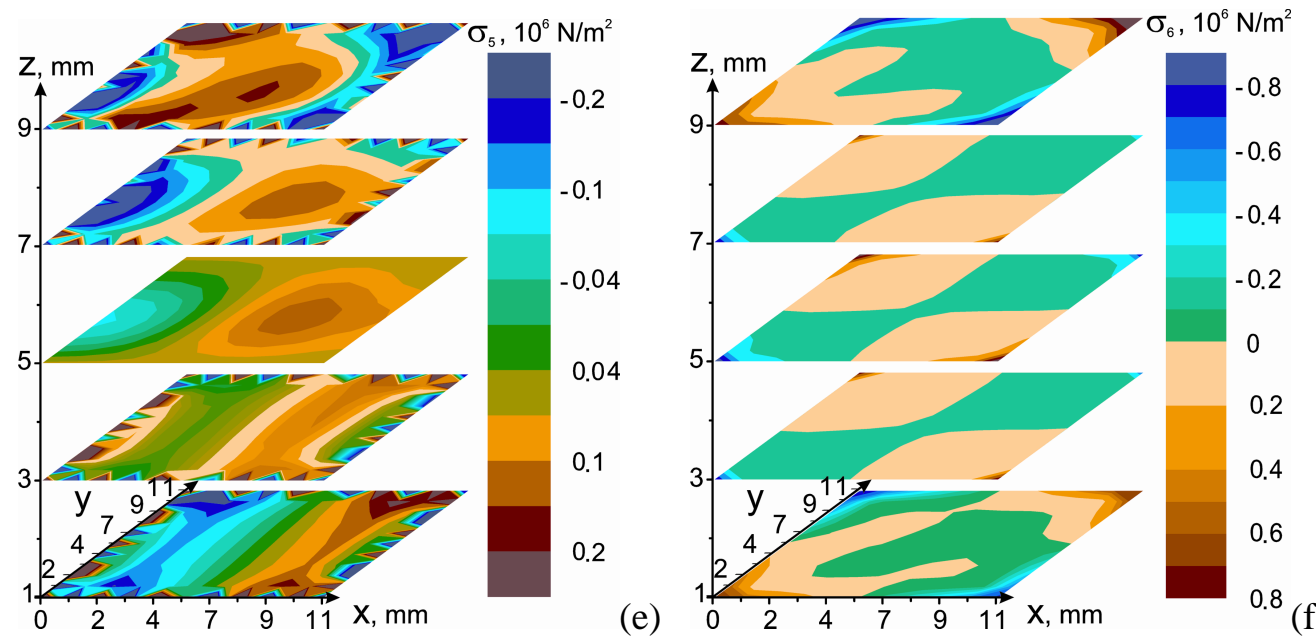

Fig. 11. Reconstructed distribution of the stress tensor components inside the glass sample: (a) $\sigma_{3}$, (b) $\sigma_{2}$, (c) $\sigma_{1}$, (d) $\sigma_{4}$, (e) $\sigma_{5}$ and (f) $\sigma_{6}$. 
tical piezooptic experiment, which does not take the distribution of mechanical stresses into account, the piezooptic coefficients determined experimentally differ from their actual values at least by $8.5 \%$. Of course, only complete reconstruction of the stress field inside a sample and a consistent consideration of the stress tensor components at each sample point would allow one to obtain a unique value of the piezooptic coefficient. In our case the latter is equal to $n_{0}^{3}\left(\pi_{11}-\pi_{12}\right)=5.52 \times 10^{-12} \mathrm{~m}^{2} / \mathrm{N}$.

In the present study we have considered the simplest case of isotropic glass only. However, in the case of anisotropic media different components of piezooptic tensor can differ as notably as by some orders of magnitude. Hence, the existence of the stress tensor components $\sigma_{1}, \sigma_{2}, \sigma_{4}, \sigma_{5}, \sigma_{6}$ which are smaller than $\sigma_{3}$ can lead to considerable errors for the piezooptic coefficients. Perhaps, the only way out is to use the technique for reconstructing the stress field described above. This should allow eliminating all the errors caused by inhomogeneity of the stress field inside the sample under study.

\section{Conclusions}

We have revealed that enormous errors appearing in many typical piezooptic experiments are mainly caused by the friction forces that exist inside an intermediate contact layer between the upper and lower sample surfaces and the corresponding substrates, along with misalignments of mechanical loading. The friction force leads to a barrel-shaped distortion of samples and inevitable appearance of all components of the stress tensor, despite of the fact that a uniaxial pressure has been initially applied.

The method for reconstruction of such 3D stress tensor fields has been proposed that includes a polarimetric mapping technique, optical retardation simulations based on the integral Jones matrix approach and a simple mechanical model. As a result of a number of natural approximations, the stress field for the cubic isotropic glass sample has been successfully reconstructed. We have also shown that reconstruction of the stress field allows achieving significant increase in the accuracy of piezooptic experiments. The corresponding errors could be reduced down to $4 \%$, which in fact represents a residual, rather apparatus than systematic error.

\section{References}

1. Grakh I I and Mozhanskaya A F, 1971. A type of mechanically anisotropic, optically sensitive material. Mekhanika Polimerov 5: 835-839.

2. Weber Y-J, 1995. Determination of internal strain by optical measurements. Phys. Rev. B 51: 12209-12215.

3. Narasimhamurty T S, Photoelastic and Electrooptic Properties of Crystals. New York: Plenum Press, (1981).

4. Slezinger I I, Alievskaya A N and Mironov Yu V, 1985. Piezooptic devices. Izmeritelnaya Tekhnika 12: 17-19.

5. Auld B A, Acoustic Fields and Waves in Solids. Malabar, FL: Krieger, (1990). 
6. Balakshii V I, Parygin V N and Chirkov L E, Physical fundamentals of acoustooptics. Moscow: Radio i Sviaz', (1985).

7. $\mathrm{Xu} \mathrm{J}$ and Stroud R, Acousto-optic Devices: Principles, Design, and Applications. New York: Wiley, (1992).

8. Shaskolskaya M P, Acoustic crystals. Moscow: Nauka, (1982).

9. Ajmera P K, Huner B, Dutta A K, and Hartley C S, 1988. Simulation and observation of infrared piezobirefringent images in diametrically compressed semiconductor disks. Appl. Opt. 27: 752-757.

10. Martynyuk-Lototska I, Mys O, Dudok T, Adamiv V, Smirnov Ye and Vlokh R, 2008. Acoustooptic Interaction in $\alpha-\mathrm{BaB}_{2} \mathrm{O}_{4}$ and $\mathrm{Li}_{2} \mathrm{~B}_{4} \mathrm{O}_{7}$ Crystals. Appl. Opt. 47: 34463454.

11. Martynyuk-Lototska I, Dudok T, Mys O and Vlokh R, 2009. Elastic, Piezooptic and Acoustooptic Properties of $\mathrm{SrB}_{4} \mathrm{O}_{7}$ and $\mathrm{PbB}_{4} \mathrm{O}_{7}$ Crystals. Opt. Mater. 31:. 660-667.

12. Mys O, Adamiv V, Martynyuk-Lototska I, Burak Ya and Vlokh R, 2007. Piezooptic and acoustic properties of $\mathrm{KLiB}_{4} \mathrm{O}_{7}$ crystals. Ukr.J.Phys.Opt. 8: 138-142.

13. Dixon R W, Cohen M G, 1966. A new technique for measuring magnitudes of photoelastic tensors and its application to lithium niobate. Appl. Phys. Lett. 8: 205-207.

14. Dixon R W, 1967. Photoelastic properties of selected materials and their relevance for application to acoustic light modulators and scanners. J. Appl. Phys. 38: 51495153.

15. Peng H J, Wong S P, and Ho H P, 2004. Measurement of orientation dependent stress-optic coefficient of GaAs single crystals. Appl. Phys. Lett. 84: 1829-1831.

16. Aben H, Errapart A, and Ainola L, 2006. On real and imaginary algorithms of optical tensor field tomography. Proc. Estonian Acad. Sci. Phys. Math. 55: 112-127.

17. Hammer H. and Lionheart W R B, 2005. Reconstruction of spatially inhomogeneous dielectric tensors through optical tomography. J. Opt. Soc. Am. A 22: 250-255.

18. Puro A É and Karov D D, 2007. Tensor field tomography of residual stresses. Opt. Spectr. 103: 678-682.

19. Aben H, Errapart A, Ainola L and Anton J, 2005. Photoelastic tomography for residual stress measurement in glass. Opt. Eng. 44: 093601.

20. Vlokh R, Krupych O, Kostyrko M, Netolya V and Trach I, 2001. Gradient thermooptical effect in $\mathrm{LiNbO}_{3}$ crystals. Ukr. J. Phys. Opt. 2: 154-158.

21. http://lzos.ru/produktsiya-nashego-predpriyatiya/

22. http://hypertextbook.com/facts/2005/glass.shtml

23. Bayda E N , Some spatial problems of the theory of elasticity. Leningrad: Izd. LGU, (1983).

\footnotetext{
Анотація. В роботі запропонований метод реконструкиії $3 D$ тензорного поля напружень 3 використанням поляриметричного картографування, моделювання оптичної різниці фаз на основі підходу інтегральних матриць Джонса і відповідної механічної моделі. Показано, щуо реконструкція поля напружень суттєво підвищує точність n'єзооптичного експерименту, тоді як відповідні похибки можуть бути зменшеними практично до апаратних.
} 